\title{
Comparison of appropriateness of cholesterol testing in general practice with the recommendations of national guidelines: an audit of patient records in 20 general practices
}

\author{
Trudy van der Weijden, Albina Dansen, Berna J Schouten, J Andre Knottnerus, Richard \\ PTM Grol
}

\begin{abstract}
Objective- To compare the profiles of those patients selected by general practitioners for measurement of serum cholesterol with the recommended profiles for opportunistic cholesterol testing described in the national practice guidelines published by the Dutch College of General Practitioners.

Design- Retrospective audit of general practitioners' records.

Materials- Practice records of 3577 adult patients systematically sampled from 20 general practices.

Main measures- With criteria set by the national guidelines, the proportion of patients per practice (a) for whom cholesterol testing would be considered justified, and (b) for whom cholesterol testing would be considered unjustified, and the proportion of patients within each of these groups who had had a cholesterol measurement recorded.
\end{abstract}

Results- Cholesterol tests were performed on 415 (11.7\%) of the 3577 patients. National guidelines on the management of hypercholesterolaemia state that a positive cardiovascular risk profile is an indication for cholesterol measurement. Just under one fifth (668) of the patients in this study were recorded as having a positive cardiovascular risk profile, but only $31 \%$ of these had had their cholesterol measured. Of the patients without recorded evidence of a positive cardiovascular risk profile cholesterol had been measured in $8 \%$. Restricting the analyses to the age group 18-65 $(n=3060)$ of whom $12.5 \%$ had a positive risk profile, did not improve the results. In practices with a computerised information system $37 \%$ of patients with recorded evidence of a positive cardiovascular risk profile had had their cholesterol measured.

Conclusions- Cholesterol testing was not targeted as selectively as recommended by the national guidelines. The major problem was failure to test those likely to benefit. Improving the targeting of cholesterol measurements would undoubtedly increase the workload of general practitioners. If the national guidelines are to have an effect on health promotion the first step must be to increase the proportion of patients with positive cardiovascular risk profiles who get their cholesterol tested. A major factor in successfully selecting cases seems to be that practices are equipped with a computerised medical information system. (Quality in Health Care 1996;5:218-222)

Keywords: cholesterol testing, national guidelines, computers

\section{Introduction}

Consensus meetings on cholesterol management in various countries, the introduction of new medication to lower cholesterol, and the portable blood testing device for cholesterol measurement, have prompted the Dutch College of General Practitioners to set guidelines for the management of hypercholesterolaemia in general practice. These guidelines, which were published in November $1991,{ }^{1}$ are based on opportunistic cholesterol testing of patients with individual positive risk profiles for coronary heart disease; the use of dietary interventions is the first line intervention recommended for those with high cholesterol concentrations. Drug treatment is recommended only for those who do not respond adequately (box). These guidelines developed for general practitioners are similar to those published in 1987 for Dutch physicians $^{2}$ except that they recommend restricting opportunistic targeting of cholesterol measurement to those aged 18-65. Despite the publication of guidelines there is no consensus about the screening strategy for use in general practices, ${ }^{3}$ and controversy surrounds the investigation and management of hypercholesterolaemia, ${ }^{4-6}$ problems with which had been noted before the publication of the guidelines in $1991 .^{7}$ In our view the most important aspect of the management of hypercholesterolaemia is the first step - the targeted approach to investigation - selecting cases. More information on targeting is needed to assess both the feasibility of the guidelines in daily practice and the need for an implementation strategy. This study compares the targeting of cholesterol screening by Dutch general practitioners in routine practice with the recommendations on selecting cases set out in the guidelines. 


\section{Methods}

GENERAL DESIGN

The study was based on 32 general practitioners who worked in 20 general practices. Practices were eligible for inclusion in the study if $(a)$ there was at least one full time general practitioner; $(b)$ they employed a practice assistant; (c) patients' records were stored in a way that allowed efficient and reproducible sampling and collection; and (d) all general practitioners in the practice were willing to participate.

In each practice patients with and without a risk profile for coronary heart disease recorded in their practice records were identified through a retrospective audit. The following data were collected in a standardised form from the records of selected patients: demographic data including age and sex; cardiovascular risk factors indicating the need for cholesterol testing (box); evidence of measurement of cholesterol, high density lipoprotein, low density lipoprotein, or triglycerides in the two year period 1 October 1990 to 30 September 1992. If at least one of the six risk factors mentioned in the guidelines was recorded somewhere in the notes the patient was considered to have a positive risk profile.

\section{Cholesterol guidelines of the Dutch College of General Practitioners}

\section{SELECTING CASES}

Cases to be selected; men and women aged 18-65, with one of the following risk factors; coronary heart disease in patient's history, signs of familial

hypercholesterolaemia (xanthoma, xanthelasma/arcus senilis before the age of 40), familial hyperlipidaemia in a relative, coronary heart disease in a sibling or parent $<60$, hypertension, and diabetes mellitus.

Patients whose only risk factor is smoking are advised to stop smoking first. DIAGNOSIS

Diagnosis of hypercholesterolaemia: the mean of three cholesterol tests $>6.5$ $\mathrm{mmol} / \mathrm{l}$, determined in a period of six weeks. Measurement of high density lipoprotein and triglycerides only when drugs to lower cholesterol are being considered.

MANAGEMENT

The patient should be referred in cases of familial hypercholesterolaemia.

$<6.5 \mathrm{mmol} /$ lgeneral advice about low fat diet.

6.5-10.0 Prescribe dietary treatment, with support, for six months. Referral to dietician if unsuccessful.

$<10.0$ Consultation with a specialist. Consider drugs to lower cholesterol after six or 12 months of dietary treatment if: serum cholesterol is $6.5-7.9 \mathrm{mmol} / 1$ and there are $\geq$ two risk factors (as defined under selecting cases); serum cholesterol is $8.0-10.0 \mathrm{mmol} / 1$ and there is at least one risk factor.

Target concentration for serum cholesterol is $6.5 \mathrm{mmol} / 1$.
Table 1 Percentage of different possibilities in the targeting of cholesterol testing

\begin{tabular}{|c|c|c|c|c|}
\hline \multirow{2}{*}{$\begin{array}{l}\text { Practice } \\
\text { sample size }\end{array}$} & \multicolumn{2}{|c|}{ Cholesterol tested } & \multicolumn{2}{|c|}{ Cholesterol not tested } \\
\hline & $\begin{array}{l}\text { Risk } \\
\text { profile + }\end{array}$ & $\begin{array}{l}\text { Risk } \\
\text { profile - }\end{array}$ & $\begin{array}{l}\text { Risk } \\
\text { profile + }\end{array}$ & $\begin{array}{l}\text { Risk } \\
\text { profile - }\end{array}$ \\
\hline $218^{\star}$ & 4.1 & 5.1 & 9.6 & 81.2 \\
\hline 231 & 8.7 & 7.8 & 15.2 & 68.4 \\
\hline 209 & 1.0 & 1.0 & 23.0 & 75.1 \\
\hline 223 & 3.1 & 4.5 & 17.0 & 75.3 \\
\hline $215^{\star}$ & 5.6 & 10.2 & 7.9 & 76.3 \\
\hline 186 & 2.7 & 7.5 & 11.3 & 78.3 \\
\hline $117^{\star}$ & 6.0 & 2.6 & 7.7 & 83.8 \\
\hline $116^{\star}$ & 6.0 & 7.8 & 5.2 & 81.0 \\
\hline 135 & 3.7 & 10.4 & 11.1 & 74.8 \\
\hline 134 & 4.5 & 4.5 & 9.0 & 82.1 \\
\hline 205 & 7.3 & 5.4 & 16.6 & 70.7 \\
\hline 209 & 10.0 & 5.7 & 22.0 & 62.2 \\
\hline $212^{\star}$ & 5.7 & 7.1 & 9.4 & 77.8 \\
\hline $216^{\star}$ & 4.2 & 8.8 & 9.7 & 77.3 \\
\hline 167 & 6.6 & 4.8 & 16.8 & 71.9 \\
\hline 185 & 1.6 & 4.9 & 19.5 & 74.1 \\
\hline 179 & 5.0 & 10.6 & 11.2 & 73.2 \\
\hline $152^{\star}$ & 4.0 & 1.6 & 12.5 & 82.2 \\
\hline 134 & 4.5 & 8.2 & 18.7 & 68.7 \\
\hline 134 & 7.5 & 13.4 & 11.2 & 67.9 \\
\hline
\end{tabular}

^ Practices with a computerised medical information system.

SAMPLING AND EXTRACTION OF DATA

Guided by previous research, ${ }^{89}$ a sample of $10 \%$ of patients aged over 18 in each practice was estimated to be sufficient for the purpose of this study. However, the sample size was increased to $16.5 \%$ to allow for those who did not respond to the request for use of data. (In The Netherlands there is a statutory requirement for informed consent from patients to allow data to be extracted from their notes, even if the data remain anonymous and are for the audit, if the data are handled by anyone other than the patient's physician or the practice assistant.)

To show a difference of at least $20 \%$ in the incidence of cholesterol testing between patients with and without identified cardiovascular risk factors - assuming that $5 \%$ of the patients not at risk are tested and a ratio of patients with risk to those without risk is $1: 3$, and applying an $\alpha$ of $5 \%$ and a power (1- $\beta$ ) of $80 \%$ - a minimum sample size of 136 per practice is needed. We aimed to select 6000 patients' records from the 20 practices and, for reasons of feasibility, we set a maximum of 400 records per practice.

In the computerised practices, every nth patient ( $\mathrm{n}$ depended on size of the practice population) was systematically sampled ${ }^{10}$ from the alphabetical list of patients' records, with a standard query procedure of the computerised patient recording system. This query procedure dealt with the selection of the target group, systematic sampling, and the production of lists and labels that identified the patients included in the study.

In the non-computerised practices sampling was manual. To cover an entire practice population, the planned number of patients' charts to be sampled was spread over the different filing system units. The first chart was taken randomly at the front of each filing system unit. Every fourth eligible patient was included in the sample until the calculated number of records per filing system unit had been selected. 
All sampled patients were sent a letter from their general practitioner asking for consent to use data from their notes. In three practices reminders were sent to non-responders to get the minimum number of 136 patients per practice.

The data were extracted by two medical students trained in auditing records.

\section{STATISTICAL ANALYSIS}

The average proportion of recorded cardiovascular risk factors used as criteria for targeting cholesterol testing in each practice as well as the variation in recording risk factors between practices was calculated with medians and interquartile ranges because of skewed distribution. Differences in the recording of risk profiles were analysed separately for practices with and without computerised systems for recording medical information by unpaired two tailed $t$ test and Levene's test (significance level $\mathrm{P}=0.05$ ).

As well as the number of cholesterol tests done, the degree to which cholesterol tests were targeted at those with positive risk profiles was measured by cross table analysis with the following criteria: at least one serum cholesterol value recorded in the two year audit period; and at least one of the six risk factors mentioned in the guidelines (box) in the notes.

The level of targeting cholesterol testing in each practice was expressed as percentages of justified and unjustified tests (table 3 ): justified tests were defined as (A/all patients) $\times 100$; unjustified testing as (B/all patients) $\times 100$; patients unjustifiably not tested as (C/all patients) $\times 100$; and justifiably not tested as (D/all patients) $\times 100$. The performance scores were also analysed relatively as justified testing considering the patients with a positive risk profile $(\mathrm{A} /(\mathrm{A}+\mathrm{C}) \times 100)$, and justified non-testing considering the patients without a positive risk profile $(D /(B+D) \times 100)$. We separately analysed the age range 18-65 for which the case finding criteria were valid.

The level of targeting of cholesterol tests in the at risk groups was also calculated separately for the subgroups of practices with and without computerised medical information systems. The difference in performance between these subgroups was tested for significance by unpaired two tailed $t$ test (significance level $\mathrm{P}=0.05$ ) taking the proportions per practice as a unit of analysis.

Table 2 Distribution of the recording of positive risk factors for coronary heart disease in 3577 patients, expressed in median percentage per practice (quartiles are given to show variation between practices)

\begin{tabular}{lcl}
\hline & Median & $\begin{array}{l}\text { Interquartile } \\
\text { range }\end{array}$ \\
\hline $\begin{array}{l}\text { Coronary heart disease in patient's } \\
\text { history }\end{array}$ & 5.3 & $4-9$ \\
$\begin{array}{l}\text { Signs of familial } \\
\text { hypercholesterolaemia }\end{array}$ & 0.0 & $0-0$ \\
$\begin{array}{l}\text { Familial hyperlipidaemia in a } \\
\text { relative }\end{array}$ & 0.0 & $0-1$ \\
$\begin{array}{l}\text { Coronary heart disease in sibling } \\
\text { or parent < 60 y }\end{array}$ & 0.9 & $0-3$ \\
$\begin{array}{l}\text { Hypertension } \\
\text { Diabetes mellitus }\end{array}$ & 10.1 & $8-16$ \\
& 3.0 & $2-4$
\end{tabular}

Table 3 Classification of different possibilities during targeting of cholesterol testing, and the results presented as the mean (SD) percentage per practice

\begin{tabular}{llll}
\hline & $\begin{array}{l}\text { Risk profile } \\
\text { positive }\end{array}$ & $\begin{array}{l}\text { Risk profile not } \\
\text { positive }\end{array}$ & Total \\
\hline $\begin{array}{c}\text { Cholesterol } \\
\text { tested }\end{array}$ & A 5.1 (2.3) & B 6.6 (3.2) & $\begin{array}{l}\text { A + B 11.7 } \\
(4.4)\end{array}$ \\
$\begin{array}{c}\text { Cholesterol not } \\
\text { tested }\end{array}$ & C 13.2(5.0) & D 75.1 (5.7) & \\
$\begin{array}{c}\text { Total } \\
\text { A + C 18.3 }\end{array}$ & & $\begin{array}{l}3577 \\
\text { patients }\end{array}$ \\
\hline
\end{tabular}

\section{Results}

CHARACTERISTICS OF THE PARTICIPATING GENERAL PRACTITIONERS AND PATIENTS

Table 1 shows the raw data for all practices. The mean (SD) age of the 32 participating general practitioners, of whom five were women, was 41 (7) years. Their working experience was 11 (8) years. Ten were working alone, the rest in association with at least one other general practitioner. The size of the practice population was 3348 (1774) patients, and the number of patients per general practitioner was 2081 (618). Twelve of the 20 practices were computerised in some form, but in only seven practices (11 general practitioners) was medical information on specific patients computerised.

A total of 6310 patients was selected through the sampling process (mean (SD) 315 (84) a practice). But informed consent was obtained from only 3950 (mean (SD, range) $65 \%$ (8\%, 49\%-81\%); mean (SD) 197 (39) per practice). The results are based on $3577(90 \%(4 \%))$ patients (179 (39) per practice) that contacted their general practitioner during the two year audit period.

The patients sampled from each of the 20 practices had similar demographic characteristics. Just under half $(46 \%)$ were men. Their mean (SD) age was 45 (16) years and most $87 \%(6 \%)$ were between 18 and 65 .

\section{RECORDING CARDIOVASCULAR RISK FACTORS \\ (TABLE 2)}

Individual risk factors most often recorded in patients' notes were hypertension and coronary heart disease; these were noted respectively in $10.1 \%$ and $5.3 \%$ of patient records. General practitioners with a computerised medical information system recorded hypertension significantly less often than general practitioners who used handwritten records. But, on the other hand, significantly more variation between practices in the number of people with recorded cardiovascular risk profiles was noted in practices that used handwritten records than for those in practices with computerised patients' records.

The mean (SD) percentage of patients in each practice with a positive risk profile (at least one of the six cardiovascular risk factors mentioned in the national guidelines) was $18.3 \%(5.4 \%)$. The same figure for those aged $18-65$, the age group discussed in the national guidelines, was $12.5 \%(3.7 \%)$.

CHOLESTEROL MEASUREMENTS

The mean (SD) percentage of patients per practice who had had cholesterol measured 
during the two year audit period was $11.7 \%$ (4.4\%). This included $9.6 \%$ (3.8\%) of patients aged 18-65. Although the positive risk profiles were evenly distributed between men and women in all age categories, in the 18-65 age group men were tested more often than women (men 59\%, women $41 \%$ ), and women aged 65 and over were twice as likely to have had their cholesterol measured than men of the same age (men 33\%, women 67\%).

\section{TARGETING OF CHOLESTEROL TESTING (TABLES}

$1,3)$

For a mean (SD) of $13.2 \%(5.0 \%)$ of patients in each practice there was no record of a cholesterol test although, according to the national guidelines, it should have been measured because of a positive risk profile. Cholesterol tests had been done, although unnecessarily according to the national guidelines, in patients without a positive risk profile, in $6.6 \%(3.2 \%)$ of patients per practice.

We found $668(18.3 \%)$ patients with evidence of a positive cardiovascular risk profile recorded in their notes, of whom $31 \%$ (SD 11\%) per practice had appropriately had their cholesterol measured $(\mathrm{A} /(\mathrm{A}+\mathrm{C}) .100)$. Of the patients without a positive cardiovascular risk profile (D/(B+D.100), 92\% (SD 4\%) per practice had appropriately not had cholesterol measured. Similar results were obtained when the analysis was restricted to patients in the 18-65 age group.

EFFECT OF COMPUTERISED PATIENTS' RECORDS We looked for differences in the appropriateness of the cholesterol measurement between practices with and without computerised medical information systems. The mean proportion of patients with a positive risk profile who had a cholesterol measurement recorded in their notes was $37 \%$ in practices with computerised medical information systems and $28 \%$ in practices without such equipment $(\mathrm{P}=0.05)$. The differences in unjustified and justified lack of testing between practices with and without computerised records were also significant $(\mathrm{P}=0.002$ for both comparisons).

\section{Discussion}

We found 415 patients who had had their cholesterol measured in the two year period. But of those with a positive risk profile according to the national guidelines (668 patients) only one third (31\%) had had their cholesterol measured, indicating that many people are not being targeted and may therefore not receive treatment to lower cholesterol. Use of a computerised medical information system seems to improve targeting of cholesterol tests.

The random samples give good insight into both doing too much and too little. We found that targeting of cholesterol tests in absolute numbers (and in percentage of the total) consists mainly of justified non-testing, so even a general practitioner who never tests any patients may show good performance. Therefore, relative performance scores, as in the subgroup of patients with a positive risk profile, give better insights into accuracy of selecting cases.

Recent comparable audits of patients' records from the United States reported that between $55 \%$ and $67 \%$ of adults had had a cholesterol value recorded, ${ }^{81112}$ but in this study of Dutch general practice we found that cholesterol was measured in only $12 \%$ of the patients. This reflects the less restrictive character of United States guidelines compared with Dutch ones; the United States guidelines recommend that all adults aged 20-70 be screened.

\section{METHODOLOGICAL POINTS TO BE CONSIDERED}

WHEN INTERPRETING THIS STUDY

Underrecording is a serious limitation for general practice audits that are based on patients' notes, particularly for details of history and advice given to patients. ${ }^{13}$ Underrecording of family history of coronary heart disease has been reported elsewhere. ${ }^{11}$ Underrecording in this audit seemed particularly relevant for three risk factors (signs of familial hypercholesterolaemia, the presence of coronary heart disease in a sibling or parent under 60 years old, and familial hyperlipidaemia in relatives). But as these three risk factors are unlikely to contribute to the histories of many patients (familial hypercholesterolaemia has been estimated to affect about one in $500^{14}$ ) our overall findings on the appropriateness of cholesterol measurement would probably not have changed much if these risk factors had been recorded properly.

The 32 general practitioners that took part in this study are in many respects (sex and list size) representative of Dutch general practitioners, but they are slightly younger and more likely to be working in group practices. Both these characteristics are reported to be factors associated with the likelihood of adopting practice guidelines. ${ }^{15}$ Thus this audit may overestimate adherance to guidelines. It does, nevertheless, give good insight into barriers that limit adherence to national guidelines, as barriers that inhibit motivated general practitioners are to be expected in others.

The difference in targeting cholesterol testing between practices with and without computerised notes could not be explained by the amount of recording of risk factors. It could be explained by the ease of accessibility of data. Obviously there may be subtle differences in the approach to practice between general practitioners working with and without this kind of computerised medical information system.

More insight is needed into other basic requirements for following preventive guidelines, one of which might be a higher level of evidence of effectiveness of the guidelines. The various cholesterol guidelines have been contradictory and controversial throughout the years. The ongoing debate about which high risk groups benefit most from cholesterol screening seems to need clarification.

We conclude that despite publication of guidelines for management of hypercholestero- 
laemia for all Dutch physicians in 1987 and for Dutch general practitioners in 1991 there remains considerable variation between practices in the aptness of cholesterol measurement. There is a clear need to improve the selection of those people for whom a cholesterol test is likely to be of benefit. The group that lose the most from the present situation, which is at best only semitargeted, are those with positive risk profiles who have not had a cholesterol measurement. These people outnumber those who are tested but for whom, according to the guidelines, cholesterol measurement is inappropriate. Thus our efforts should be directed at increasing cholesterol testing in those with positive profiles. Doing this will increase the workload of general practitioners; in fact at least one in every eight adults has a positive risk profile and is in the 18-65 age group, and so should be tested. The burden on the general practitioner goes beyond a simple test, as for all those in whom cholesterol is raised repeated tests will be needed; some will require dietary advice, and some monitored drug treatment.

We are grateful to Dr Tomas Spenser for his critical advice and support.

1 Binsbergen JJ van, Brouwer A, Drenth BB van, Haverkort AFM, Prins A, Weijden $T$ van der. NHG-standaard
Cholesterol. (Cholesterol guidelines of the Dutch College of General Practitioners.) Huisarts Wet 1991;34:551-7.

2 Cholesterol consensus. Heart Bulletin 1987:1(suppl):1-64.

3 Weijden $\mathrm{T}$ van der. Wetenschappeliike achtergronden bij de NHG-standaard cholesterol. Een literatuuroverzicht. (Scientific background of the cholesterol guidelines. A entific background of the cholesterol guid

4 Smith GD, Pekkanen J. Should there be a moratorium on the use of cholesterol lowering drugs? BMF 1992;304:431-4

5 Dunnigan MG. The problem with cholesterol. No light at the end of this tunnel? BMf 1993;306:1355-6.

6 Law MR, Thompson SG, Wald NJ. Assessing possible hazards of reducing serum cholesterol. BMF 1994;308:373-9.

7 Weijden T van der, Hutten JBF, Brandenburg BJ, Gro RPTM, Velden $\mathrm{K}$ van der. Cholesterol management in Dutch general practice. A comparison with national guidelines. Scand f Prim Health Care 1994;12:281-8.

8 Hudson JW, Keefe CW, Hogan AJ. Cholesterol measurement and treatment in community practices. Fam Pract 1990;31:139-44

9 Otradovec K, Blake RL, Parker BM. An assessment of the practice of preventive cardiology in an academic health center. F Fam Pract 1985;21:125-9.

10 Weijden $T$ van der, Höppener P, Schouten B. Computerized sampling techniques. F Fam Pract 1995;40:123.

11 McBride PE, Pacala JT, Dean J, Plane MB. Primary care residents and the management of hypercholesterolemia Am ₹Prev Med 1990;6:71-6.

12 Robinson MK, DeHaven MJ, Wallace JB, Fost T. Hypercholesterolemia: case finding in family practice. South Med 1992;85:1091-5.

13 Goldstein JL, Brown MS. Familial hypercholesterolemia. In: Scriver Beaudet CR, Sly WS, Volle DJ, eds. The metabolic basis of inherited disease. 6th ed. New York: McGrawHill, 1989: 215-51.

14 Rethans JJ, Martin E, Metsemakers J. To what extent do clinical notes by general practitioners reflect actual medical performance? A study using simulated patients. Brf Gen Pract 1994;44:153-6.

15 Grol R. National standard setting for quality of care in general practice: attitudes of general practitioners and response to set of standards. Br $\mathcal{F ~ G e n ~ P r a c t ~ 1 9 9 0 ; 4 0 : 3 6 1 - 4 . ~}$ 